\title{
NUMERICAL ANALYSIS OF THE SEISMIC BEHAVIOR OF TUNNELS CONSTRUCTED IN LIQUEFIABLE SOILS
}

\author{
F. KHOSHNOUDIAN ${ }^{\mathrm{i})}$ and I. SHAHROUR ${ }^{\mathrm{ii})}$
}

\begin{abstract}
This paper includes a numerical study of the seismic behavior of tunnels constructed in liquefiable soils. It is carried out using finite element modeling based on the $(u-p)$ formulation (displacement for the solid phase and pore-pressure for the fluid phase). The behavior of the soil material is modeled using a cyclic elastoplastic constitutive relation involving both isotropic and kinematic hardening. The paper is composed of three parts. The first part includes a brief presentation of the numerical model used in this study; the second concerns the analysis of a reference example; the last part presents a parametric study of the influence of both loading and soil characteristics on the seismic response of tunnels constructed in saturated soils.
\end{abstract}

Key words: cyclic, elastoplastic, finite element, liquefaction, pore-pressure, saturated, seismic, tunnels (IGC: E2)

\section{INTRODUCTION}

Analysis of case histories such as the Niigata and Hokkaido-Nansei-Oki earthquakes (Okamoto, 1984; Tokimatsu et al., 1994) showed that earthquake-induced liquefaction caused serious damage to underground structures. These observations were confirmed by shaking table tests, which showed that light underground structures constructed in liquefiable soils suffer major uplift damage due to the pore-pressure built-up induced by seismic loading (Koseki et al., 1997; Koseki et al., 1998).

Little work is reported on the numerical analysis of the seismic behavior of tunnels constructed in liquefiable soils. In this paper, we present a study of this problem using the $(u-p)$ formulation (displacement for the solid phase and pore-pressure for the fluid phase) proposed by Zienkiewicz et al. (1980). The behavior of the soil material is described using a cyclic elastoplastic constitutive relation, which can reproduce liquefaction of loose soils under undrained loading paths. After a description of the numerical model, we present the analysis of a reference example followed by a study of the influence of some parameters on the seismic response of tunnels constructed in saturated soils.

\section{NUMERICAL MODELING}

The seismic behavior of tunnels constructed in saturated soils is analyzed using the finite element program PECPLAS which offers facilities for the analysis of earthquake problems (Shahrour, 1992; Khoshravan,
1995; Shahrour et al., 1995; Ousta, 1998). The fluidskeleton interaction is modeled using the $(u-p)$ formulation which is based on neglecting the relative fluid acceleration in the equilibrium equations and in the Darcy flow law (Zienkiewicz et al., 1980).

\section{Constitutive Relation}

The soil behavior is described by means of the cyclic elastoplastic constitutive relation MODSOL (Shahrour and Chehade, 1992; Shahrour et al., 1995), which is based on the concept of bounding surface (Dafalias and Popov, 1975; Mroz and Pietruszczak, 1983). MODSOL includes two families of surfaces (Fig. 1). The first one, called the bounding surface, controls the domain of vir-

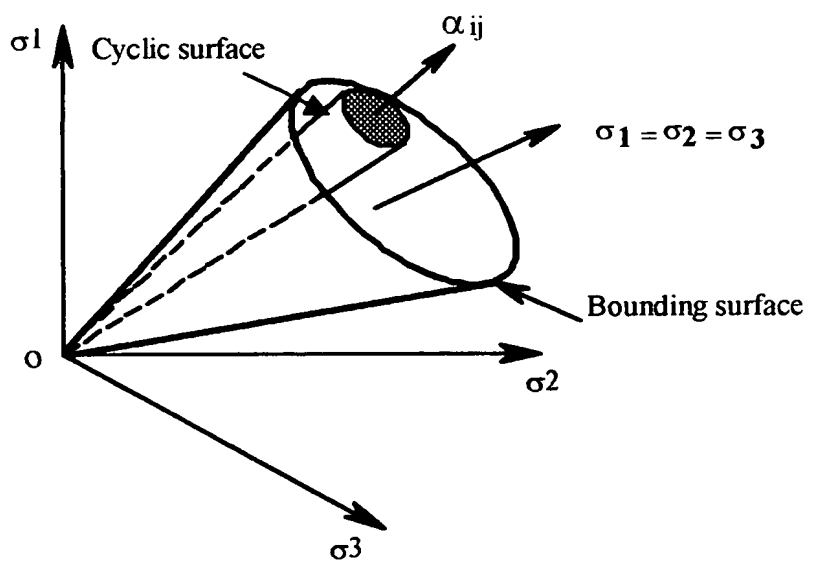

Fig. 1. Yield surfaces used in the constitutive relation MODSOL

i) Laboratoire de Mécanique de Lille (CNRS URA 1441) Université des Sciences et Technologies de Lille-EUDIL, 59655 Villeneuve d'Ascq cedex, France.

ii) ditto, Isam.Shahrour@eudil.fr.

Manuscript was received for review on September 6, 2001.

Written discussions on this paper should be submitted before July 1, 2003 to the Japanese Geotechnical Society, Sugayama Bldg. 4F, Kanda Awaji-cho 2-23, Chiyoda-ku, Tokyo 101-0063, Japan. Upon request the closing date may be extended one month. 
gin loading, while the second, called the cyclic surface, encloses the domain of elasticity. The bounding surface involves isotropic hardening, while the cyclic surface involves both isotropic and kinematic hardening.

According to the elastoplastic theory, the total strain increment $(d \varepsilon)$ is assumed to be the sum of the elastic strain increment $\left(d \varepsilon^{\mathrm{e}}\right)$ and the plastic strain increment $\left(d \varepsilon^{\mathfrak{p}}\right)$ :

$$
d \varepsilon=d \varepsilon^{\mathrm{e}}+d \varepsilon^{\mathrm{p}} .
$$

The elastic part is completely described using the bulk modulus $(K)$ and the shear modulus $(G)$ which are assumed to be stress-dependent. The mathematical formulation of the elastic part is given by:

$$
\begin{aligned}
& d \sigma_{\mathrm{ij}}=\left(K-\frac{2}{3} G\right) d \varepsilon_{\mathrm{kk}}^{\mathrm{e}} \delta_{\mathrm{ij}}+2 G d \varepsilon_{\mathrm{ij}}^{\mathrm{e}} \\
& K=K_{0}\left(\frac{p_{\mathrm{m}}}{p_{\mathrm{a}}}\right)^{\mathrm{n}} \frac{1}{1-\frac{K_{0}}{3 G_{0}} n\left(q / p_{\mathrm{m}}\right)^{2}} \quad G=G_{0}\left(\frac{p_{\mathrm{m}}}{p_{\mathrm{a}}}\right)^{\mathrm{n}} \\
& p_{\mathrm{m}}=\frac{\sigma_{\mathrm{ii}}}{3} \quad q=\sqrt{\frac{3}{2} s_{\mathrm{ij}} s_{\mathrm{ij}}} \quad s_{\mathrm{ij}}=\sigma_{\mathrm{ij}}-p_{\mathrm{m}} \delta_{\mathrm{ij}}
\end{aligned}
$$

where $p_{\mathrm{m}}$ and $q$ represent the effective mean stress and the deviatoric stress, respectively; $K_{0}, G_{0}$ and $n$ are constitutive parameters; $p_{\mathrm{a}}$ is a reference pressure.

The determination of the plastic strain increment requires the definition of a yield function $(f)$ and plastic potential $(g)$. According to the theory of plasticity, plastic strains are generated if the state of stress attempts to move beyond the yield surface. Mathematically speaking this condition can be written as:

$$
f=0 \quad \text { and } \quad d f=\frac{\partial f}{\partial \sigma_{\mathrm{ij}}} d \sigma_{\mathrm{ij}}>0 .
$$

In this case, the plastic strain increment is determined from the plastic potential $(g)$ as follows:

$$
d \varepsilon_{\mathrm{ij}}^{\mathrm{p}}=d \lambda \frac{\partial g}{\partial \sigma_{\mathrm{ij}}}
$$

where $d \lambda$ is a positive scalar of proportionality, which is called the plastic consistency parameter. It is determined from the consistency condition $(d f=0)$.

In the following, we present briefly the mathematical formulation of the yield function $(f)$, the gradient of the plastic potential $(\partial g / \partial \sigma)$ and the hardening rule for both the bounding and cyclic mechanisms.

The expression of the bounding yield surface $\left(f_{\mathrm{m}}\right)$ is given by:

$$
\begin{aligned}
& f_{\mathrm{m}}=q-M_{\mathrm{f}} p_{\mathrm{m}} R_{\mathrm{m}} \\
& M_{\mathrm{f}}=\frac{6 \sin \varphi}{3-\sin \varphi \sin 3 \theta} \\
& \theta=\frac{1}{3} \sin ^{-1}\left(-\frac{27 J_{3}}{2 q^{3}}\right) \quad J_{3}=\frac{s_{\mathrm{ij}} s_{\mathrm{jk}} S_{\mathrm{ki}}}{3} \quad-\frac{\pi}{6} \leq \theta \leq \frac{\pi}{6}
\end{aligned}
$$

where $\varphi$ and $\theta$ designate the friction angle and the Lode angle, respectively. This surface coincides with the Mohr-
Coulomb failure surface for triaxial condition. The hardening function $R_{\mathrm{m}}$ depends on the deviatoric plastic strain $\left(\varepsilon_{\mathrm{d}}^{\mathrm{p}}\right)$ as follows:

$$
\begin{aligned}
& R_{\mathrm{m}}=\frac{\varepsilon_{\mathrm{d}}^{\mathrm{p}}}{a+\varepsilon_{\mathrm{d}}^{\mathrm{p}}} \\
& \varepsilon_{\mathrm{d}}^{\mathrm{p}}=\int\left(\frac{2}{3} d e_{\mathrm{ij}}^{\mathrm{p}} d e_{\mathrm{ij}}^{\mathrm{p}}\right)^{1 / 2} d e_{\mathrm{ij}}^{\mathrm{p}}=d \varepsilon_{\mathrm{ij}}^{\mathrm{p}}-\frac{1}{3} d \varepsilon_{\mathrm{kk}}^{\mathrm{p}} \delta_{\mathrm{ij}}
\end{aligned}
$$

where $a$ is a constitutive parameter.

The flow rule for the bounding surface is defined by the gradient of the plastic potential function $\left(g_{\mathrm{m}}\right)$ in the form:

$$
\begin{aligned}
& \frac{\partial g_{\mathrm{m}}}{\partial \sigma_{\mathrm{ij}}}=\frac{\exp \left(-\alpha_{0} \varepsilon_{\mathrm{d}}^{\mathrm{p}}\right)}{3 M_{\mathrm{c}} p_{\mathrm{m}}}\left(M_{\mathrm{c}}-\frac{q}{p_{\mathrm{m}}}\right) \delta_{\mathrm{ij}}+\frac{1}{M_{\mathrm{c}} p_{\mathrm{m}}} \cdot \frac{s_{\mathrm{ij}}}{q} \\
& M_{\mathrm{c}}=\frac{6 \sin \varphi_{\mathrm{cv}}}{3-\sin \varphi_{\mathrm{cv}} \sin 3 \theta} .
\end{aligned}
$$

Here, $\varphi_{\mathrm{cv}}$ denotes the characteristic angle which corresponds to zero plastic volume change; $\alpha_{0}$ is an adjusting parameter which allows for stabilization of the volume change for high values of the deviatoric plastic strain.

Numerical simulations presented in this paper were performed assuming a circular conical shape form for the cyclic yield surface. This assumption is made to simplify both the mathematical formulation of the constitutive relation and its numerical integration, but it does not guarantee the non-intersection of the cyclic yield and bounding surfaces. The expression of the cyclic yield surface $\left(f_{\mathrm{c}}\right)$ is given by:

$$
\begin{aligned}
& f_{\mathrm{c}}=q^{1}-p^{1} R_{\mathrm{c}} \\
& p^{1}=\sigma_{\mathrm{ij}} \alpha_{\mathrm{ij}} \quad q^{1}=\sqrt{\left(s_{\mathrm{ij}}^{1} s_{\mathrm{ij}}^{1}\right)} \quad s_{\mathrm{ij}}^{1}=\sigma_{\mathrm{ij}}-p^{1} \alpha_{\mathrm{ij}}
\end{aligned}
$$

$R_{\mathrm{c}}$ denotes the hardening function which depends on the deviatoric plastic strain induced since the last stress reversal $\left(\varepsilon_{\mathrm{dc}}^{\mathrm{p}}\right)$ :

$$
R_{\mathrm{c}}=\frac{\varepsilon_{\mathrm{dc}}^{\mathrm{p}}}{a+\varepsilon_{\mathrm{dc}}^{\mathrm{p}}}
$$

$\alpha_{\mathrm{ij}}$ denotes the unit tensor along the axis of the cyclic yield surface. Its variation is governed by the following expressions:

$$
\begin{aligned}
& d \alpha_{\mathrm{ij}}=d \lambda c_{\mathrm{c}}\left(1-A \frac{q}{M_{\mathrm{f}} p_{\mathrm{m}} R_{\mathrm{m}}}\right) R_{\mathrm{c}} s_{\mathrm{ij}}^{1} \\
& A=\left\{\begin{array}{rll}
1 & \text { if } \quad s_{\mathrm{ij}} s_{\mathrm{ij}}^{1} \geq 0 \\
-1 & \text { if } \quad s_{\mathrm{ij}} s_{\mathrm{ij}}^{1}<0
\end{array}\right.
\end{aligned}
$$

$d \lambda$ is the plastic consistency parameter; $c_{\mathrm{c}}$ is a constitutive parameter.

The flow rule for the cyclic surface is given by the gradient of the cyclic plastic potential $\left(g_{\mathrm{c}}\right)$ in the form: 


$$
\begin{aligned}
& \left(\frac{e^{-\alpha_{\alpha} \varepsilon_{\mathrm{f}}^{\mathrm{p}}}}{3 M_{\mathrm{c}} p}\left(M_{\mathrm{c}}-\frac{q}{p}\right) \delta_{\mathrm{ij}}+\frac{1}{M_{\mathrm{c}} p} \cdot \frac{s_{\mathrm{ij}}}{q}\right. \\
& \text { if } \frac{\partial g_{\mathrm{m}}}{\partial \sigma_{\mathrm{k} 1}} d \sigma_{\mathrm{k} 1} \geq 0 \\
& \frac{\partial g_{\mathrm{c}}}{\partial \sigma_{\mathrm{ij}}}=\mid \begin{array}{l}
\frac{e^{-\alpha_{\mathrm{c}} \mathrm{p}_{\mathrm{p}}^{\mathrm{p}} e^{-\alpha_{\alpha} \mathrm{p}_{\mathrm{p}}^{\mathrm{p}}}}}{M_{\mathrm{c}} p^{1}}\left(M_{\mathrm{c}}-\frac{3 q^{1}}{p^{1}}\right) \alpha_{\mathrm{ij}} \\
\quad+\frac{3}{2 M_{\mathrm{c}} p^{1} q^{1}}\left(s_{\mathrm{ij}}^{1}-\left(s_{\mathrm{k} 1}^{1} \alpha_{\mathrm{k} 1}\right) \alpha_{\mathrm{ij}}\right)
\end{array} \\
& \text { if } \frac{\partial g_{\mathrm{m}}}{\partial \sigma_{\mathrm{k} 1}} d \sigma_{\mathrm{k} 1}<0
\end{aligned}
$$

$\alpha_{\mathrm{c}}$ is a constitutive parameter which controls the variation of the volumetric strain during cyclic loading.

This constitutive relation was verified on centrifuge tests performed within VELACS project (Shahrour et al., 1995).

\section{Numerical Procedure}

Numerical simulations are performed in two stages (Khoshnoudian, 1999). The first stage concerns the determination of the initial state (stresses and hardening parameters of the constitutive relation MODSOL). This phase is carried out in two steps using a static drained finite element modeling: the first step concerns the computation of gravity stresses existing before the tunnel excavation; the second step corresponds to the tunnel construction, which is modeled by deactivation of excavated elements and activation of the liner elements.

The second stage concerns the seismic analysis. It is carried out with the following boundary conditions (Fig. 2a):

(i) the base of the soil mass is assumed rigid and impervious;

(ii) the water table is assumed to coincide with the ground surface $(p=0)$;

(iii) periodic conditions are imposed at lateral boundaries for both pore-pressure and displacements.

The seismic loading is modeled by a harmonic acceleration imposed at the base of the soil mass.

\section{REFERENCE EXAMPLE}

\section{Description of the Reference Example}

The reference example concerns a circular tunnel with a diameter $D=9 \mathrm{~m}$, constructed at a depth of $H=1.8 D$ in a saturated soil, whose density and permeability are equal to $\rho_{\mathrm{s}}=1960 \mathrm{~kg} / \mathrm{m}^{3}$ and $k=0.001 \mathrm{~m} / \mathrm{s}$ (Fig: 2a). The finite element mesh used in the numerical simulation is composed of 608 isoparametric 8-node elements (Fig. 2b).

The set of parameters used for the description of the soil behavior is presented in Table 1 . It was determined from triaxial tests performed on Nevada sand within VELACS project (Khoshravan, 1995). Figure 3 presents the simulation of a cyclic undrained triaxial path with this set of parameters. It can be observed that the model predicts an increase in the pore-pressure which leads to soil liquefaction after four cycles.

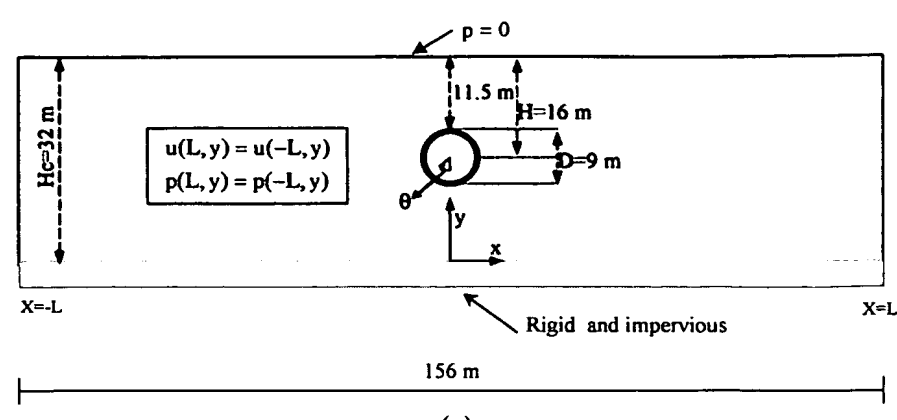

(a)

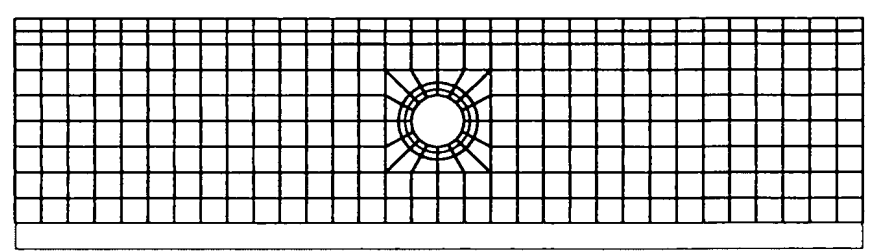

(b)

Fig. 2. (a) Boundary conditions and properties of the reference example, (b) Finite element mesh used for the reference example

Table 1. Set of parameters used in the reference example

\begin{tabular}{c|c|c|c|c|c|c|c|c}
\hline$K_{0}$ & $G_{0}$ & $n$ & $\varphi$ & $\varphi_{\mathrm{cv}}$ & $\alpha_{0}$ & $a$ & $c_{\mathrm{c}}$ & $a_{\mathrm{c}}$ \\
\hline $27.7 \mathrm{MPa}$ & $18.3 \mathrm{MPa}$ & 0.5 & $38^{\circ}$ & $30^{\circ}$ & 8 & $9.4 \mathrm{E}-4$ & 0.024 & 100 \\
\hline
\end{tabular}

The behavior of the liner is assumed to be linear-elastic with the following properties: Young's modulus $E_{\mathrm{r}}=$ $21000 \mathrm{MPa}$, Poisson's ratio $v_{\mathrm{r}}=0.25$ and density $\rho_{\mathrm{r}}=$ $2400 \mathrm{~kg} / \mathrm{m}^{3}$. Its thickness is equal to $0.4 \mathrm{~m}$.

The seismic loading is applied at the base of the soil mass as a harmonic acceleration involving eight cycles: $\ddot{u}_{\mathrm{g}}(t)=A_{\mathrm{g}} \sin (2 \pi f t)$. The loading amplitude and frequency are equal to $A_{\mathrm{g}}=0.1 \mathrm{~g}$ and $f=1 \mathrm{~Hz}$. The latter is close to the fundamental frequency of a homogeneous soil mass having the mean value of the initial elastic modulus of the reference case $(f 1=0.75 \mathrm{~Hz})$.

\section{Results and Discussion}

Figure 4 presents the variation of the excess pore-pressure normalized by the initial vertical effective stress in the soil mass $\left(\sigma_{v 0}^{\prime}\right)$. In the proximity of the ground surface, we observe a sharp increase of the pore-pressure up to values close to that of the initial vertical effective stress $\left(\sigma_{v 0}^{\prime}\right)$, which indicates the occurrence of liquefaction in this zone. Near the base of the soil mass, a progressive increase of the pore-pressure is observed up to values which remain smaller than the vertical effective stress, this phase is followed by dissipation of the excess pore-pressure. An extension of the liquefied zone towards the base of the soil mass is observed during the application of the seismic loading.

Figure 5 presents a comparison between the excess pore-pressure obtained in the reference example and that of pressure induced in a free-field condition. It can be observed that the presence of the tunnel mainly affects the variation of the pore-pressure in the zone located under 

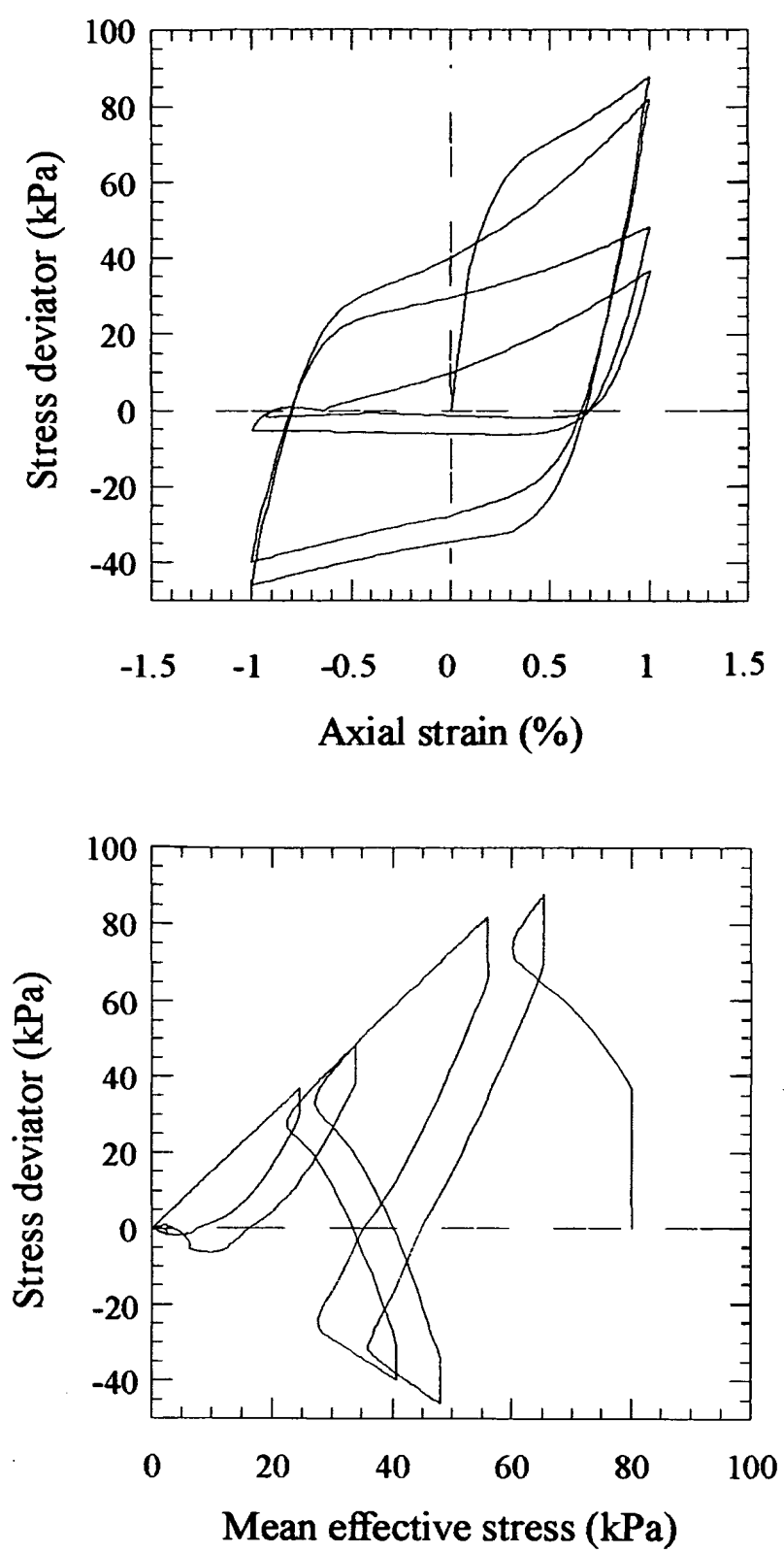

Fig. 3. Numerical simulation of a triaxial undrained test with the set of parameters used in the reference example (cell pressure= $80 \mathrm{kPa})$

the tunnel. It induces a reduction of about $25 \%$ of the pore-pressure in this zone. This result agrees well with experimental observations reported by Koseki et al. (1997).

Figure 6 shows the profile of the vertical displacement at the ground surface. It indicates an uplift of the soil located above the liner, which results from a pore-pressure build-up induced by the seismic loading. This phenomenon also was observed in shaking table tests (Koseki et al., 1997).

The evolution of the bending moment at the crown of the tunnel is depicted in Fig. 7a. It shows an increase of the bending moment during the application of seismic loading up to $270 \mathrm{kNm}$, followed by a decrease down to $170 \mathrm{kNm}$, which presents $63 \%$ of the maximum bending moment. The envelope of the bending moment in the liner is illustrated in Fig. 7b. It indicates an important
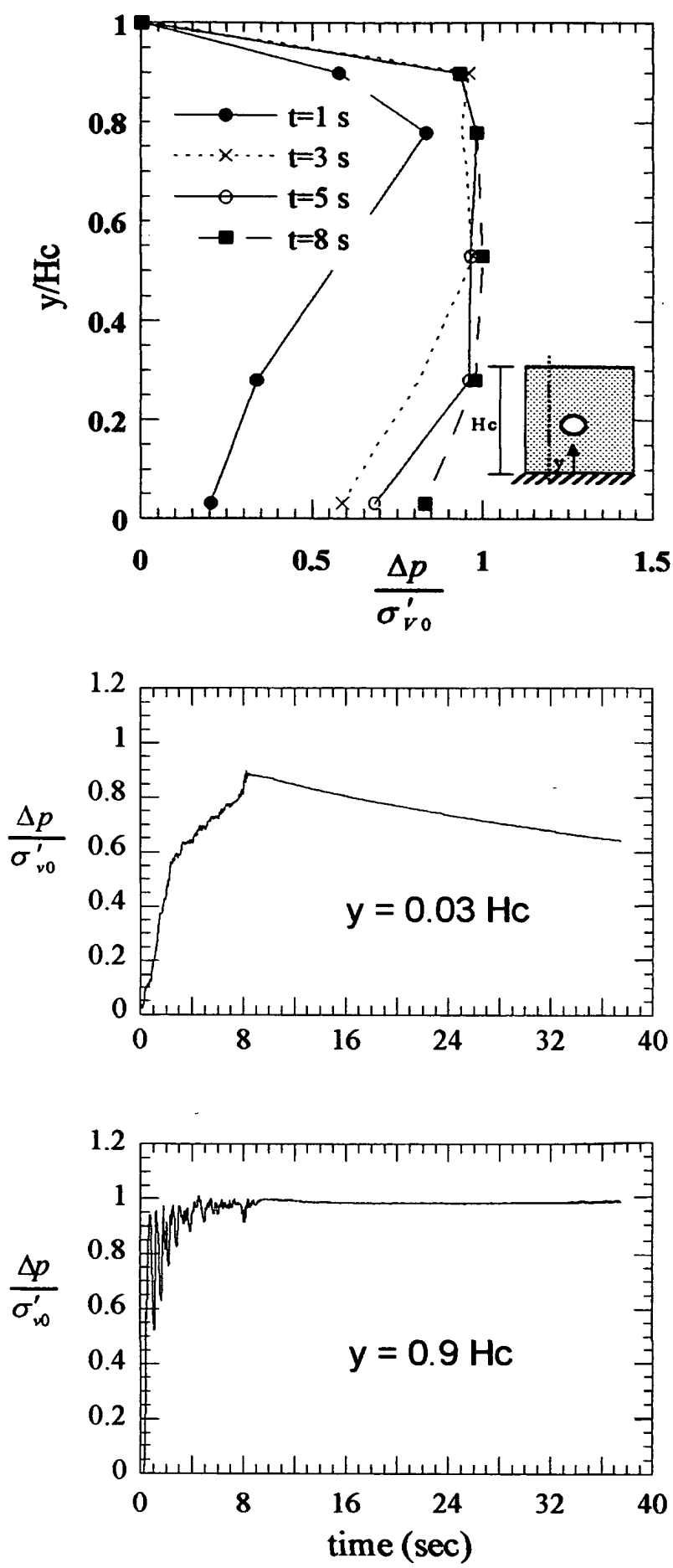

Fig. 4. Reference example/History of the pore pressure in the soil mass

variation of the bending moment in the liner with peaks in sections located at $\theta=0^{\circ}, 75^{\circ}, 180^{\circ}$ and $290^{\circ}$.

The influence of the soil saturation on the seismic response of the tunnel was investigated by comparing results obtained with saturated and dry sands, respectively (Fig. 8). Results for the dry sand were obtained from a mono phase finite element modeling (the fluid phase was neglected) performed with the effective initial state of the reference example. It can be observed that soil saturation induces an important reduction of the 

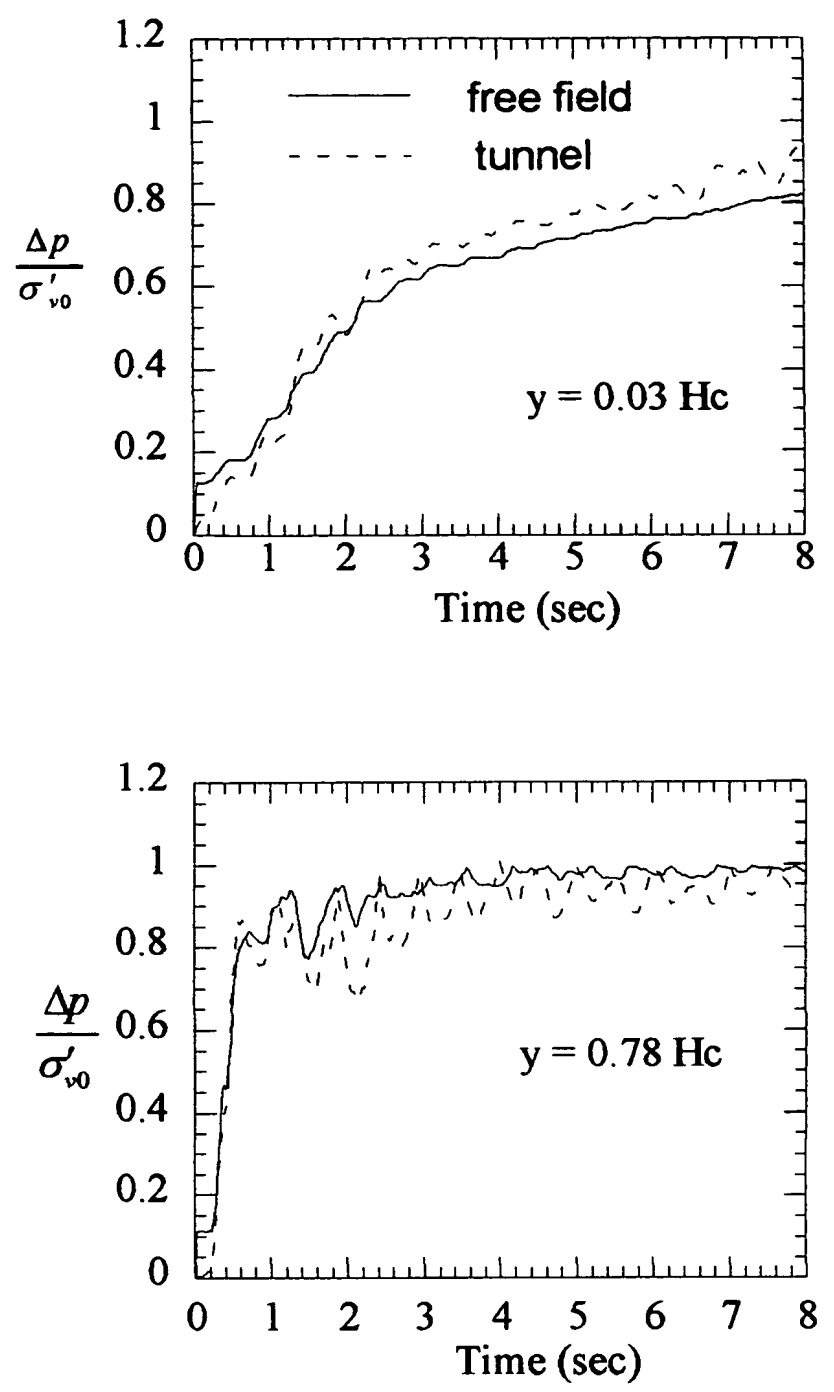

Fig. 5. Reference example/Influence of the presence of the tunnel on the pore-pressure built-up: a) Under the liner, b) Above the liner

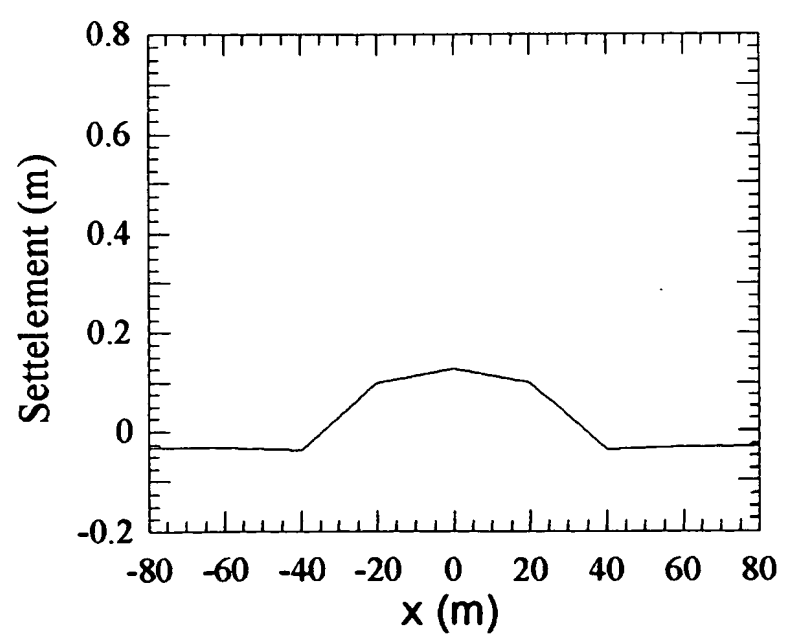

Fig. 6. Reference example/Vertical displacement at the ground surface
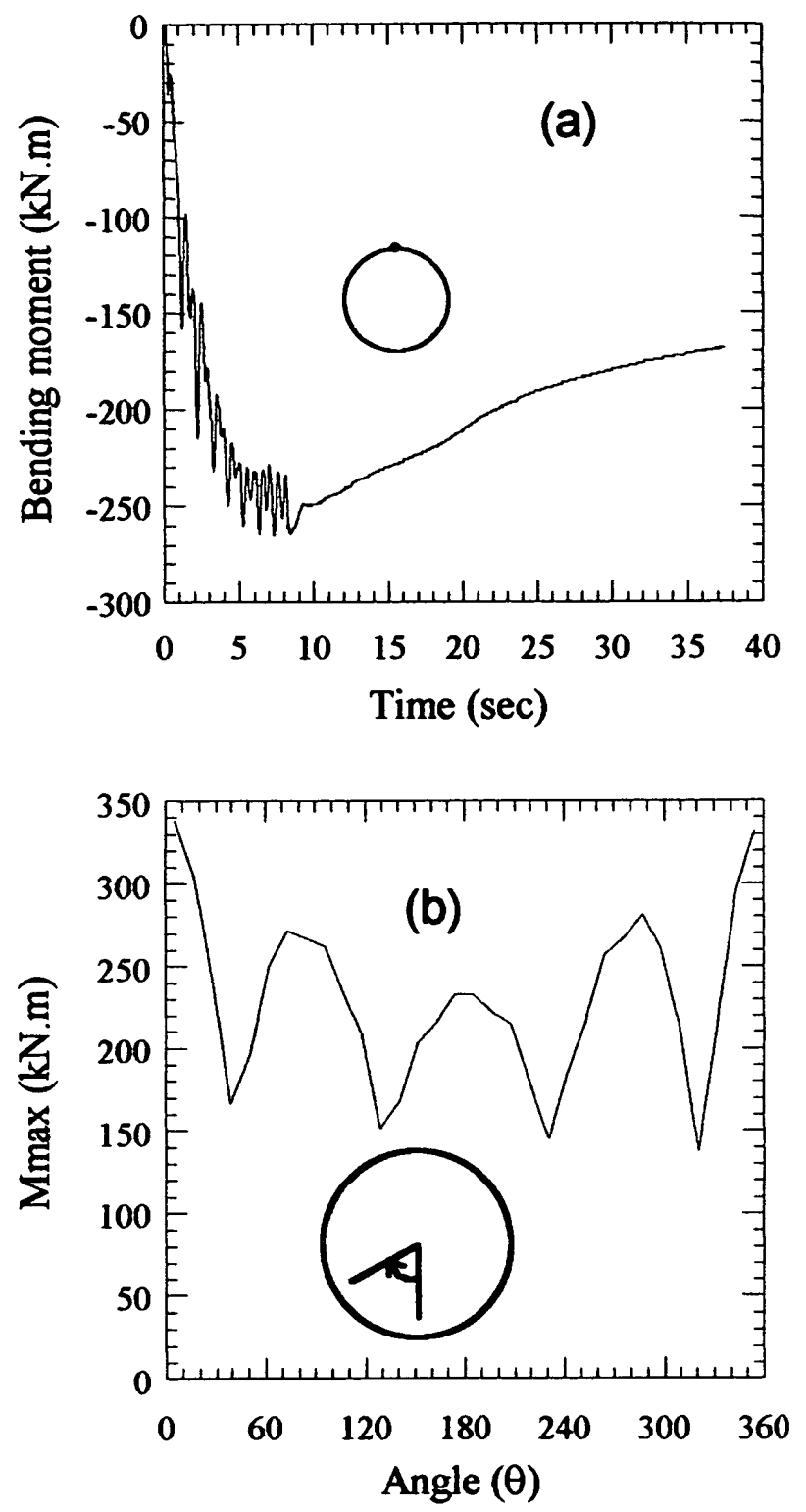

Fig. 7. Reference example/Bending moment induced by the seismic loading: a) History of the bending moment at the crown of the tunnel, b) Envelope of the bending moment in the liner

dynamic amplification as reported in Fig. 8 a (260\% for saturated soils and $600 \%$ for dry sand). This reduction results from the excess pore-pressure, which reduces the effective stresses and consequently disturbs the transmission of the acceleration in the soil mass. It can also be noted that soil saturation induces a high increase of the bending moment, which reaches about $200 \%$ of that calculated with dry sand (Fig. 8b). This amplification is due to the pore-pressure built-up, which induces an important degradation of the soil stiffness and resistance.

\section{PARAMETRIC STUDY}

This section includes an analysis of the influence of some parameters on the seismic behavior of tunnels constructed in saturated sands. 

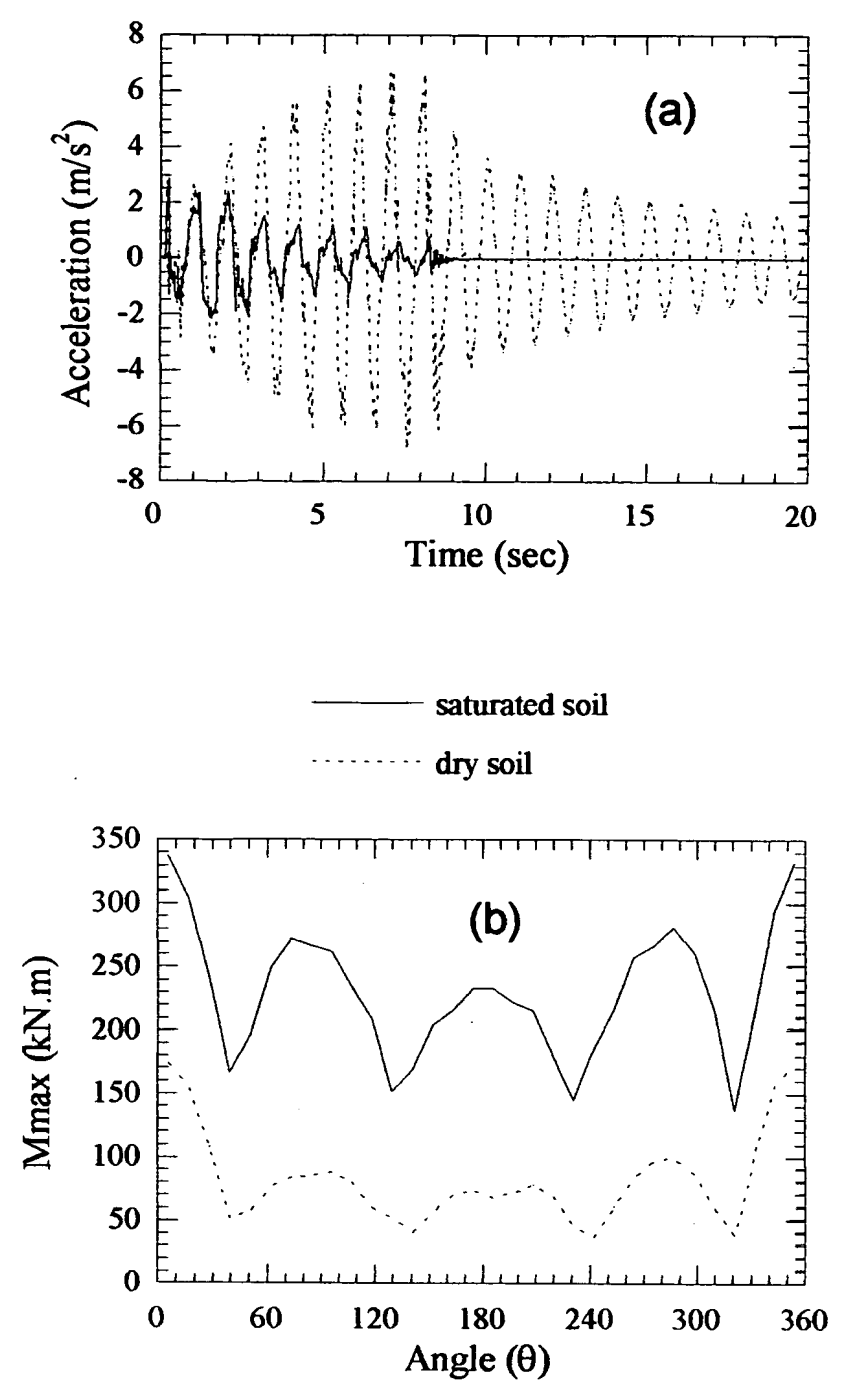

Fig. 8. Influence of the soil saturation on the seismic response of the tunnel: a) History of the acceleration at the crown of the tunnel, b) Envelope of the bending moment

\section{Influence of the Loading Frequency}

Figure 9 presents the influence of the loading frequency on the seismic response of the tunnel presented in the reference example. It can be observed that the loading frequency largely affects the excess pore-pressure in the lower part of the soil mass (Fig. 9a). The influence of the loading frequency is also important on the bending moment: the decrease of the loading frequency from $2.3 \mathrm{~Hz}$ to $1 \mathrm{~Hz}$ yields a significant increase in the bending moment, which reaches $125 \%$ in the lower part of the liner (Fig. 9b).

\section{Influence of the Loading Amplitude}

The influence of the loading amplitude on the response of the tunnel is depicted in Fig. 10. It can be observed that the augmentation of the loading amplitude from $0.1 \mathrm{~g}$ to $0.2 \mathrm{~g}$ causes an increase in the pore-pressure and an extension of the liquefied zone towards the base of the soil mass. A moderate variation of the bending moment (about 10\%) is observed with the increase of the loading frequency. This moderate influence is due to the increase
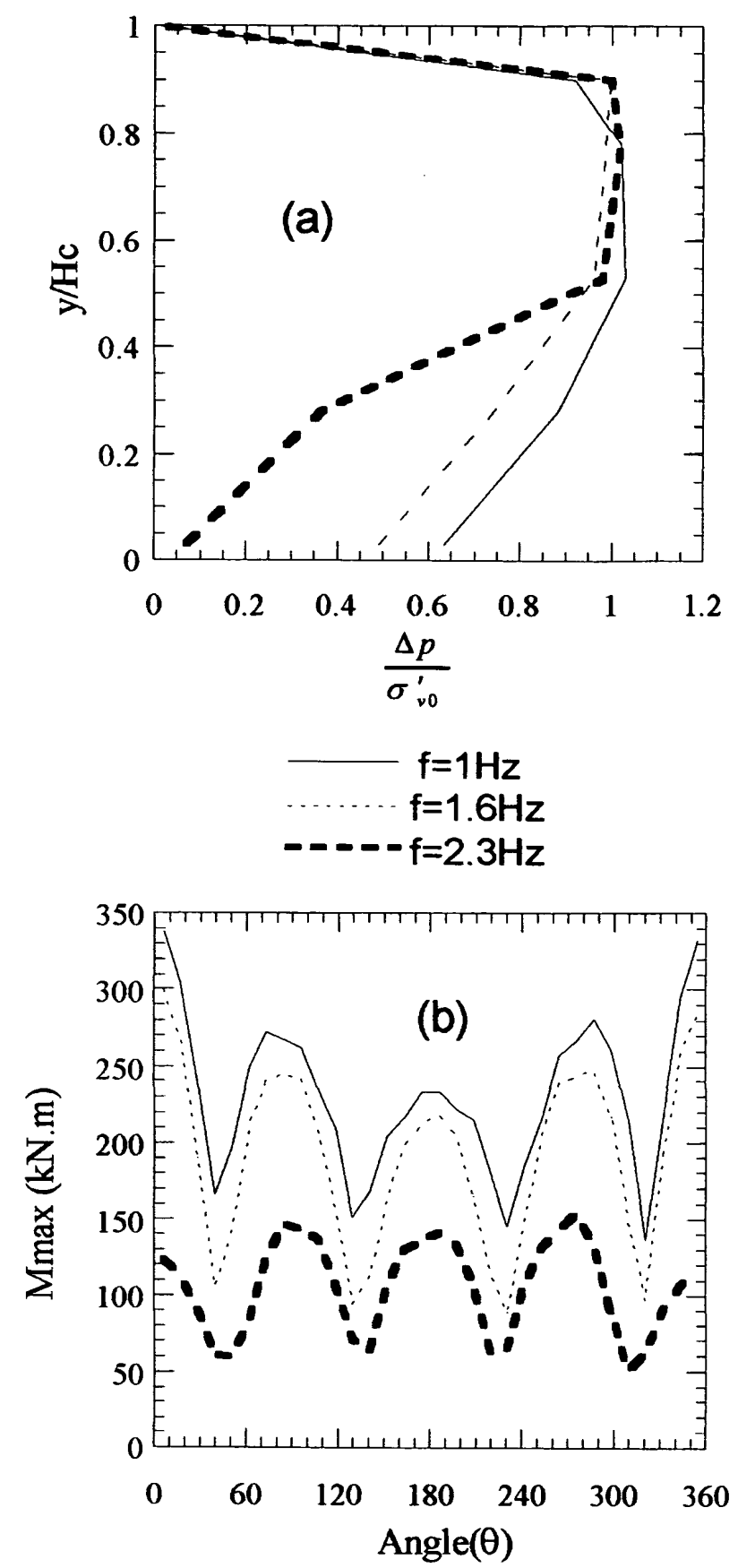

Fig. 9. Influence of the loading frequency on the seismic response of the tunnel: a) Evolution of the pore-pressure, b) Envelope of the bending moment

of pore-pressure, which attenuates the dynamic amplification in the soil mass. The same variation of the loading amplitude for a similar tunnel constructed in dry sand induces an increase of about $40 \%$ in the bending moment (Khoshnoudian, 1999).

\section{Influence of the Soil Permeability}

Figure 11 presents the influence of the soil permeability on the response of the tunnel considered in the reference case. It can be observed that the increase in the soil permeability from $0.001 \mathrm{~m} / \mathrm{s}$ to $0.008 \mathrm{~m} / \mathrm{s}$ moderately affects the pore-pressure built-up, but largely accelerates 

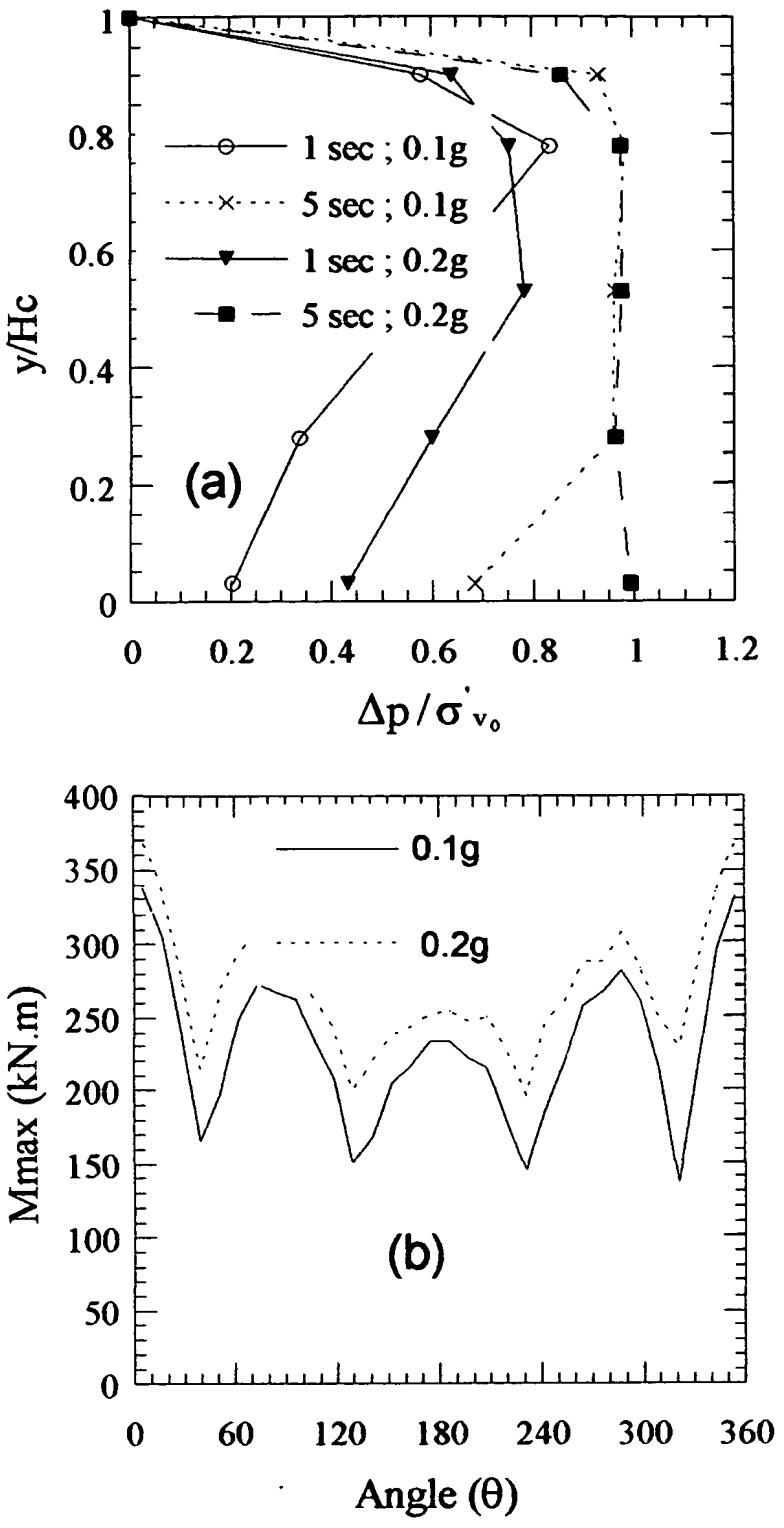

Fig. 10. Influence of the loading amplitude on the seismic response of the tunnel: a) Excess pore-pressure, b) Envelope of the bending moment

its dissipation. At $t=25 \mathrm{sec}$, the excess pore-pressure with $k=0.008 \mathrm{~m} / \mathrm{s}$ is about $50 \%$ of that obtained with $k=$ $0.001 \mathrm{~m} / \mathrm{s}$. It can also be noted that the increase of the soil permeability from $0.001 \mathrm{~m} / \mathrm{s}$ to $0.008 \mathrm{~m} / \mathrm{s}$ induces a reduction in the bending moment, which reaches about $25 \%$ in the lower part of the liner.

\section{Influence of the Soil Dilatancy}

The influence of the soil dilatancy on the seismic response of the tunnel is investigated though a variation of the characteristic angle $\varphi_{\mathrm{cv}}$ whose augmentation reduces the soil dilatancy. Figure 12 shows results obtained with two values of $\varphi_{\mathrm{cv}}$. It can be observed that the increase of the soil dilatancy by a reduction of $\varphi_{\mathrm{cv}}$ from $30^{\circ}$ to $23^{\circ}$ reduces the excess pore-pressure and decreases the bending moment in the lower part of the liner.
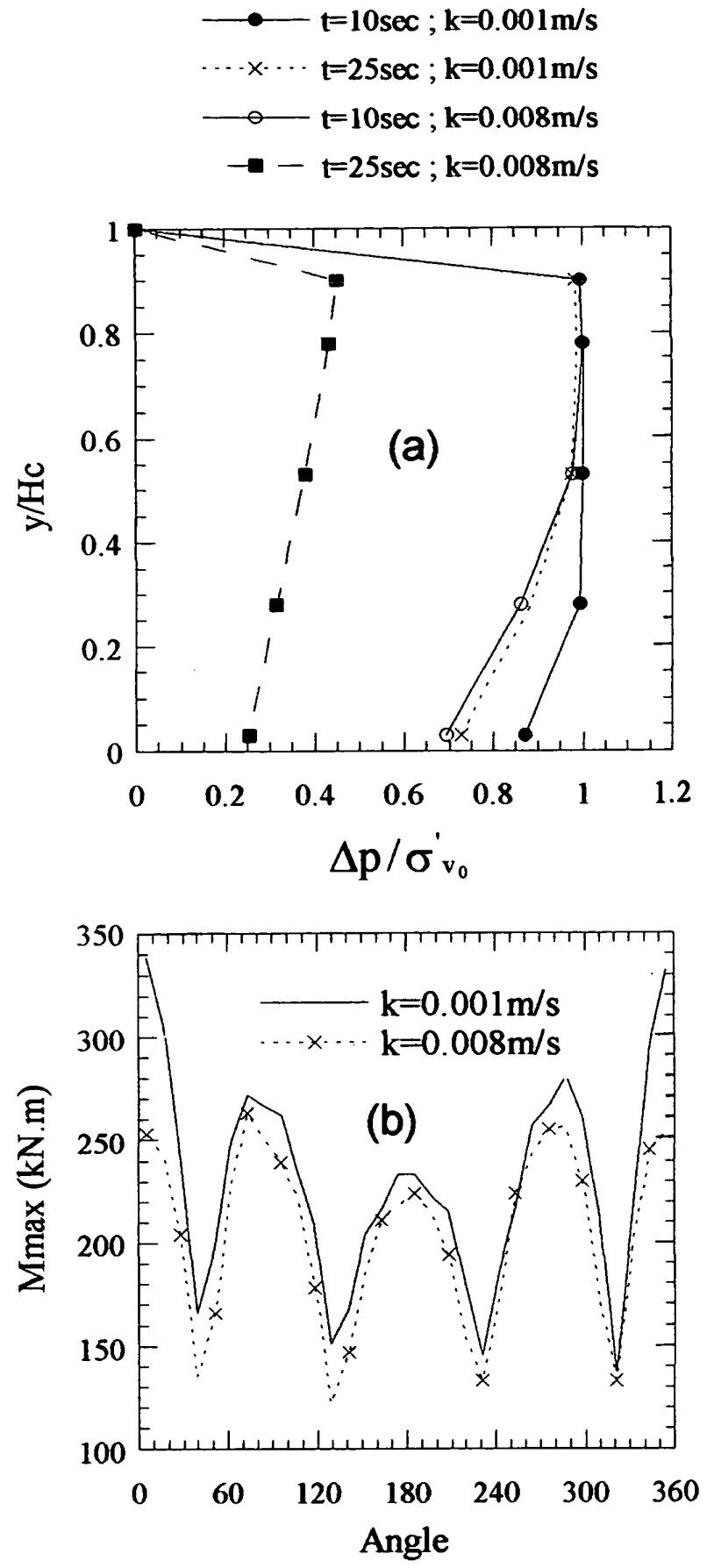

Fig. 11. Influence of the soil permeability: a) Excess pore-pressure, b) Envelope of the bending moment

\section{CONCLUSION}

This paper includes a numerical analysis of the seismic behavior of lined tunnels constructed in liquefiable soils. The study was performed using the $(u-p)$ formulation (displacement for the solid phase and pore-pressure for the fluid phase) and a cyclic elastoplastic constitutive relation for the soil behavior. Numerical simulations show that the seismic loading induces a significant increase in the pore-pressure, particularly in the upper part of the soil mass. The presence of the liner reduces the pore-pressure build-up in the soil located under the liner. As the 

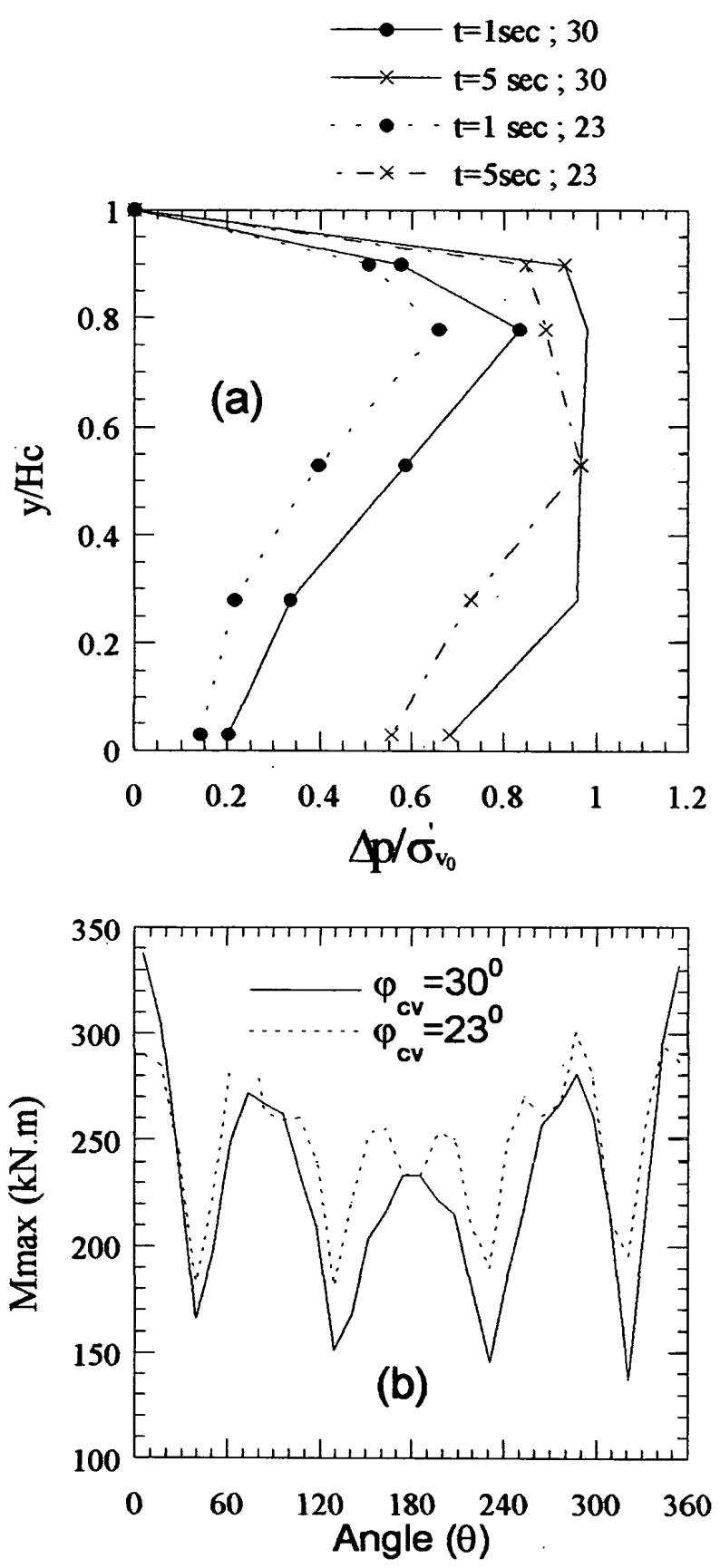

Fig. 12. Influence of the soil dilatancy on the seismic response of the tunnel: a) Excess pore-pressure, b) Envelope of the bending moment excess pore-pressure degrades the soil stiffness and resistance, it causes an important increase of the bending moment in the liner, particularly in the lower part. It also produces an uplift of the liner.

\section{REFERENCES}

1) Dafalias, Y. F. and Popov, E. P. (1975): A model of non-linearity hardening for cyclic loading, Acta Mechanica, 21, 173-192.

2) Khoshnoudian, F. (1999): Etude du comportement des tunnels sous chargement sismique, PhD Thesis, University of Sciences and Technologies of Lille, France.

3) Khoshravan, A. (1995): Problèmes des sols saturés sous chargement dynamique: Modèle cyclique pour les sols et validation sur des essais en centrifugeuse, PhD Thesis, University of Sciences and Technologies of Lille, France.

4) Koseki, J., Matsuo, O. and Koga, Y. (1997): Uplift behavior of underground structures caused by liquefaction of surrounding soil during earthquake, Soils and Foundations, 37(1), 97-108.

5) Koseki, J., Matsuo, O. and Tanaka, S. (1998): Uplift of sewer pipes caused by earthquake-induced liquefaction of surrounding soil, Soils and Foundations, 38(3), 75-87.

6) Mroz, Z. and Pietruszczak, S. (1983): A constitutive model for sand with anisotropic hardening rule, Int. J. Num. and An. Meth. in Geom., 7, 305-320.

7) Okamoto, S. (1984): Introduction to Earthquake Engng., 2nd ed., University of Tokyo Press.

8) Ousta, R. (1998): Etude du comportement sismique des micropieux, PhD Thesis, University of Sciences and Technologies of Lille, France.

9) Shahrour, I. (1992): PECPLAS: A finite element software for the resolution of earthwork problems, Actes du Colloque Int. Géotechnique and Informatique, Edition Presse ENPC, Paris, 327-334.

10) Shahrour, I. and Chehade, W. (1992): Development of a constitutive elastoplastic model for soils, XI Int. Congress on Rheology Brussels (eds. by Moldenaers, P. and Keuning, R.), Elsevier.

11) Shahrour, I., Benzenati, I. and Khoshravan, A. (1995): Validation of nonlinear coupled dynamic model on centrifuges tests of VELACS project, Numerical Models in Geomechanics, NUMOG V, ed. Balkema, Rotterdam, 269-274.

12) Tokimatsu, K., Suzuki, Y. and Tamura, S. (1994): Preliminary report on geotechnical aspects of Hokkaido-Nansei-Oki Earthquake of July, 12, 1993, Performance of Ground and Soil Structure during Earthquake, 13th Int. Conf. On SMFE, New Delhi, 75-86.

13) Zienkiewicz, C., Chang, C. T. and Bettess, P. (1980): Drained untrained, consolidating and dynamic behavior assumptions in soils: Limits of validity, Géotechnique, (30), 385-395. 